\section{STRESS AS HUMAN ELEMENT AT WORK: A SURVEY OF} FILIPINO SEAFARERS

Don Eliseo Lucero-Prisno III. University of the Philippines, Open University, The Philippines

10.1136/oemed-2014-102362.342

Objectives Seafaring entails working on board ships for a long period of time away from home. This results into various psychological experiences by the world's 1.2 million seafarers working on international commercial vessels. Filipino seafarers comprise almost $30 \%$ of the world's seafarers. The study will look at the stress management profile of Filipino seafarers including how stress is manifested and implications for prevention.

Method Questionnaires were administered to 2500 Filipino seafarer respondents representing various ranks/positions. Respondents were chosen from different manning agencies and training centres in different parts of the Philippines. Different sets of questionnaires were administered to different sectors such as management and labour. The questionnaire was divided into the following categories: socio-demographic profile, health and lifestyle, attitude towards work and family/home, work and homerelated experiences, symptoms/signs of stress, coping with work experiences, and infrastructure on board.

Results Respondents were 69\% ratings and 31\% officers mostly within the age of $25-50$ working in bulk carrier vessels and tankers. Health problems normally experienced are vision, hypertension, muscular, hearing and respiratory. 50\% drink alcohol and 20\% smoke on board. 55\% sleep well and $87 \%$ exercise. Most workers are satisfied with their jobs. Significant sources of stress are routinary nature of job, long hours of work, tension among crew, and thoughts of impending early retirement. Home-work interface elements are major sources of stress such as family concerns and careers of wives.

Conclusions Socio-psychological problems need to be addressed by developing appropriate programmes. This should be mainstreamed in the occupational health agenda for seafarers.

\section{USE OF SALIVARY BIOMARKERS TO EVALUATE RESPONSE TO A STRESS MANAGEMENT INTERVENTION}

1,2David Koh. 'Universiti Brunei Darussalam, Brunei, Brunei Darussalam; ${ }^{2}$ National University of Singapore, Singapore, Singapore

\subsection{6/oemed-2014-102362.343}

Objectives To discuss methodological issues related to using salivary biomarkers to evaluate response to a stress management intervention.

Method Findings from a study which utilised salivary biomarkers to evaluate group responses to a stress management program are discussed.

In that study, we measured responses to qigong practice as a stress intervention among 34 healthy adults.

Results Specific biomarkers studied were a stress hormone (cortisol); a surrogate marker co-released with acute stress (alpha amylase); and a marker of early physiological response to stress i.e. immune status as reflected by immunoglobulin A (IgA).

Salivary cortisol and IgA were monitored over 10 weeks in the intervention group $(\mathrm{n}=18)$ and the control group $(\mathrm{n}=16)$.

Median salivary cortisol concentrations (nmol/l) at weeks 1,6 and 10 were 4.4, 4.8, 4.3 and 4.3, 4.0, 3.3 for the control and intervention groups. Median IgA secretion rates $(\mu \mathrm{g} / \mathrm{min})$ were
$58.9,63.6$ and 67.4 for the control group and 43.8, 54.9 and 72.9 for the intervention group.

Acute response to qigong practice, measured by median salivary alpha amylase $(\mathrm{U} / \mathrm{ml})$ showed no significant change before and after a one hour session of practice (107.7 and 93.8).

Saliva collection technique, circadian rhythm and half-life of the biomarkers, and their relative concentrations in different body compartments e.g. blood and saliva, can affect the results and were taken into account in the study protocol.

Conclusions For valid interpretation of study findings, the choice of biological markers and other methodological issues have to be considered when using salivary biomarkers to evaluate response to a stress management intervention.

\section{MUNICIPAL CARTOGRAPHY OF ASBESTOS EXPOSURE IN A PARIS' SUBURB: AN ORIGINAL USE OF AN OCCUPATIONAL EXPOSURE DATABASE}

1,2 Benjamin Lysaniuk. ' UMR 8586 PRODIG/CNRS, Paris, France; ' GISCOP93, Bobigny, France

\subsection{6/oemed-2014-102362.344}

Objectives Fibrex database can be described as a board giving a correspondence between jobs and quantitative indications of occupational exposure to one or several noxious fibres. The objective of this present work is to propose a municipal index of asbestos exposure and finally map it by crossing the Fibrex database with activity sectors data of the French national institute for statistics and economic studies (INSEE) at a city scale in a northern Paris' suburb.

Method Fibrex database consists of more than 10000 data of occupational exposure to organic or inorganic fibres from natural or artificial origins. It allows a focus on asbestos fibres by giving a median value of exposure to that carcinogenic substance within a given activity sector. Knowing the distribution of the workforces by activity sectors and associating the median values of exposures of the considerate work, it is possible to envisage an exposure score at a city scale.

Results The calculated score proposes a partial but original view of the potential exposure to asbestos by territorial unit in the end of 90's. The use of city-scale data allows bringing to light the territorial heterogeneity of occupational exposures to asbestos in a Paris' suburb.

Conclusions This contribution shows an original use of an occupational exposure database ending in a city-scale mapping.

\section{SENSE OF COHERENCE AND MENTAL HEALTH AMONG SEAFARERS IN RELATION TO PHYSICAL ACTIVITY}

Vilija Malinauskiene. Lithuanian Sports University, Kaunas, Lithuania

10.1136/oemed-2014-102362.345

Objectives Seafaring is a specific occupation due to long-term isolation from the society and the family. The aim of the study was to investigate the prevalence of psychological distress (PD) and sense of coherence (SOC) among seafarers in relation to occupational and leisure time physical activity (PhA).

Method 248 seafarers, attending the Maritime Medical Centre in Klaipeda for the mandatory health examination answered the anonymous questionnaire (GHQ-12) and SOC. The mean values 